\title{
Investigating the AGN activity and black hole masses in low surface brightness galaxies
}

\author{
Smitha Subramanian ${ }^{1}$, Ramya Sethuram ${ }^{2}$, Mousumi Das ${ }^{1}$, \\ Koshy George ${ }^{1}$, Sivarani Thirupathi ${ }^{1}$ and Tushar P. Prabhu ${ }^{1}$ \\ ${ }^{1}$ Indian Institute of Astrophysics, 2nd Block, Koramangala, Bangalore - 560034, India \\ email: smitha@iiap.res.in \\ ${ }^{2}$ Shanghai Astronomical Observatory, Shanghai, China
}

\begin{abstract}
We present an analysis of the optical nuclear spectra from the active galactic nuclei $(\mathrm{AGN})$ in a sample of giant low surface brightness (GLSB) galaxies. GLSB galaxies are extreme late type spirals that are large, isolated and poorly evolved compared to regular spiral galaxies. Earlier studies have indicated that their nuclei have relatively low mass black holes. Using data from the Sloan Digital Sky Survey (SDSS), we selected a sample of 30 GLSB galaxies that showed broad $\mathrm{H} \alpha$ emission lines in their AGN spectra. In some galaxies such as UGC 6284, the broad component of $\mathrm{H} \alpha$ is more related to outflows rather than the black hole. One galaxy (UGC 6614) showed two broad components in $\mathrm{H} \alpha$, one associated with the black hole and the other associated with an outflow event. We derived the nuclear black hole (BH) masses of 29 galaxies from their broad $\mathrm{H} \alpha$ parameters. We find that the nuclear $\mathrm{BH}$ masses lie in the range $10^{5}-10^{7} \mathrm{M}_{\odot}$. The bulge stellar velocity dispersion $\sigma_{e}$ was determined from the underlying stellar spectra. We compared our results with the existing BH mass - velocity dispersion $\left(M_{\mathrm{BH}}-\right.$ $\sigma_{e}$ ) correlations and found that the majority of our sample lie in the low $\mathrm{BH}$ mass regime and below the $M_{\mathrm{BH}}-\sigma_{e}$ correlation. The effects of galaxy orientation in the measurement of $\sigma_{e}$ and the increase of $\sigma_{e}$ due to the effects of bar are probable reasons for the observed offset for some galaxies, but in many galaxies the offset is real. A possible explanation for the $M_{\mathrm{BH}}-\sigma_{e}$ offset could be lack of mergers and accretion events in the history of these galaxies which leads to a lack of BH-bulge co-evolution.
\end{abstract}

Keywords. galaxies: active, galaxies: bulges, galaxies: nuclei

\section{Introduction}

Low surface brightness (LSB) galaxies are late type spiral galaxies (Sc or Sd) that have a central disk surface brightness of $\mu_{B(0)} \geqslant 22$ to 23 mag $\operatorname{arcsec}^{-2}$ (Impey \& Bothun 1997; Impey et al. 2001). Disk LSBs are more rare and usually found in isolated environments (Bothun et al. 1993) and vary over a range of sizes. But the really large LSB galaxies, which are referred to as giant LSB (GLSB) galaxies, are generally found to lie close to the edges of voids (Rosenbaum et al. 2009). Nuclear activity is not common in LSB galaxies. This is in marked contrast to high surface brightness disk galaxies where the percentage having active galactic nuclei (AGN) can be as high as $50 \%$, depending on the mean luminosity of the sample. The most probable explanation for this low fraction is that LSB disks generally lack two structural features that facilitate gas flows and the formation of a compact object in the nucleus - bars and strong spiral arms (Bothun et al. 1997).

However, a significant fraction of bulge dominated GLSB galaxies show AGN activity (Sprayberry et al. 1995). AGN activity in bulge dominated GLSBs is not surprising as studies indicate that the growth of nuclear black holes $(\mathrm{BH})$ in galaxies is intimately linked to the growth of their bulges (Silk \& Rees 1998; Heckman et al. 2004). The 
strong correlation of black hole mass $\left(M_{\mathrm{BH}}\right)$ with bulge stellar velocity dispersion in galaxies $\left(M_{\mathrm{BH}}-\sigma_{e}\right)$ is due to this supermassive black hole (SMBH) - bugle co-evolution (Gültekin et al. 2009 and McConnell \& Ma 2013). But in LSB galaxies, their bulge velocity dispersion and disc rotation speeds suggest that they lie below the $M_{\mathrm{BH}}-\sigma_{e}$ correlation for bright galaxies (Pizzella et al. 2005). Mei et al. (2009) estimated the black hole masses of three GLSB galaxies and found them to lie close to the $M_{\mathrm{BH}}-\sigma_{e}$ correlation and the $\mathrm{BH}$ masses are found to be of the order of $\sim 10^{7} \mathrm{M}_{\odot}$. Later estimates from the study of another three GLSBs by Ramya et al. (2011) showed that their sample galaxies lie offset from the $M_{\mathrm{BH}}-\sigma_{e}$ correlation. They suggested that the bulges of their sample might be well evolved, but the $\mathrm{BH}$ masses $\left(3-9 \times 10^{5} \mathrm{M}_{\odot}\right)$ are lower than those found in bright galaxies. Morelli et al. (2012) found that the bulges of LSB galaxies have similar properties as that of the bulges of normal galaxies. It is not clearly understood where the LSB galaxies in general lie on the $M_{\mathrm{BH}}-\sigma_{e}$ correlation and also what are the possible reasons for the observed offset in a few sample galaxies. Low mass black holes in isolated LSB galaxies are are also very interesting candidates for the study of seed black holes in galaxies (Volonteri 2010).

\section{Data and analysis}

As an initial sample we selected 558 LSB galaxies from the literature (Sprayberry et al. 1995, Impey et al. 1996, Schombert et al. 1992 and Schombert et al. 1988) for which the Sloan Digital Sky Survey (SDSS) DR10 nuclear spectra are available. The emission line fluxes of these 558 galaxies are available for public in the SDSS DR10 database. The emission line fluxes are used to construct the $[\mathrm{NII}]_{6583} / \mathrm{H} \alpha$ vs $[\mathrm{OIII}]_{5007} / \mathrm{H} \beta$ BaldwinPhillips-Terlevich (BPT) (Baldwin et al. 1981) diagram. Out of 558 galaxies, 160 galaxies ( $\sim 29 \%$, with almost equal contribution from the composite and purely AGN candidates) are classified as AGN and the remaining 398 ( 71\%) galaxies as purely star forming systems. From the sample of 160 galaxies, we selected only those galaxies which have median S/N > 15 for further analysis. Thus, finally we used SDSS DR10 spectra of 115 LSB galaxies to identify sources with broad Balmer lines and hence estimate their black hole masses.

We used the pPXF (Penalized Pixel-Fitting stellar kinematics extraction) code by Cappellari \& Emsellem (2004) to obtain the best fit model for the underlying stellar population. pPXF also provides the stellar velocity dispersion, $\sigma$. The $\mathrm{H} \alpha+[\mathrm{NII}]$ doublet region of all the 115 galaxies are first fitted with three Gaussians, representing the three narrow lines of $\mathrm{H} \alpha$ and $[\mathrm{NII}]$ doublet. Then this region is again fitted including a fourth Gaussian component for the broad $\mathrm{H} \alpha$ line. The final sample with broad $\mathrm{H} \alpha$ component is selected based on the two criteria, that the the inclusion of the broad component should improve the reduced $\chi^{2}$ value by at least $5 \%$ and also the broad $\mathrm{H} \alpha$ peak flux should be at least three times larger than the residue of the fit. Thus based on the these criteria, 52 LSB galaxies were selected as galaxies with broad $\mathrm{H} \alpha$ component. After the careful examination of the fits of each of these galaxies, 28 of them showed the presence of broad $\mathrm{H} \alpha$ at significant level and 2 galaxies showed an extra broad components, which are significantly blue/red shifted from the $\mathrm{H} \alpha$ central wavelength and are hence more likely to be associated with an outflow rather than with the active black hole. The 28 galaxies, with genuine broad $\mathrm{H} \alpha$ component, are of our interest as their black hole masses can be estimated and hence check their M- $\sigma_{e}$ correlation. UGC 6614, which showed signatures of an outflow, has a clear broad $\mathrm{H} \alpha$ component along with the blue shifted outflow component. The other emission lines present in the spectra of these 30 galaxies (28 with broad $\mathrm{H} \alpha$ component and 2 with outflow signatures) were also analysed and fitted with 


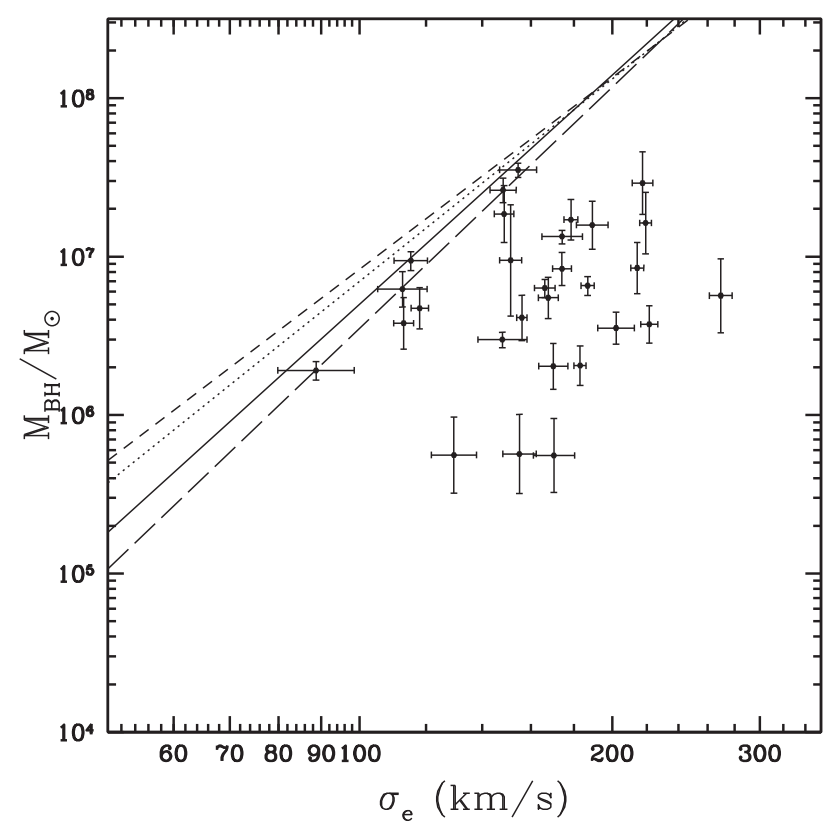

Figure 1. The M- $\sigma_{e}$ plot with broad line AGN candidates. The linear regression lines given by Tremaine et al. (2002), Ferrarese \& Merritt (2000), Gültekin et al. (2009) and McConnell \& Ma (2013) relation for late type galaxies (dashed, solid, dotted and long-dashed lines, respectively) for $M_{\mathrm{BH}}$ against $\sigma_{e}$ are also shown.

Gaussian profiles. IDL programs are used to fit the emission line profiles with Gaussian functions and the fluxes of all the emission lines are estimated.

\section{Black hole masses and the $M-\sigma_{e}$ correlation}

The virial black hole masses of 29 broad line AGN candidates are calculated using the equation given in Reines et al. (2013), using the luminosity and FWHM of broad H $\alpha$. The equation is given below. Here, the scale factor that depends on the broad line region geometry, $\epsilon$ is assumed to be 1 .

$$
\log \frac{M_{\mathrm{BH}}}{\mathrm{M}_{\odot}}=6.57+0.47 \log \frac{L_{\mathrm{H} \alpha}}{10^{42} \mathrm{erg} \mathrm{s}^{-1}}+2.06 \log \frac{\mathrm{FWHM}_{\mathrm{H} \alpha}}{10^{3} \mathrm{~km} \mathrm{~s}^{-1}}
$$

The masses estimated are in the range $5.5 \times 10^{5} \mathrm{M}_{\odot}$ to $3.5 \times 10^{7} \mathrm{M}_{\odot}$. The median mass is found to be $5.6 \times 10^{6} \mathrm{M}_{\odot}$. The median mass suggests that the LSB galaxies have black hole masses which are slightly higher than intermediate mass black holes.

The stellar velocity dispersion values obtained from pPXF were transformed to the equivalent velocity dispersion $\sigma_{e}$ at a radius of $r_{e} / 8$ in the galaxy bulge, where $r_{e}$ is the bulge exponential scale length, using the transformation equation given by Jorgensen et al. (1995). The BH mass estimates for the 29 galaxies in our sample plotted against the equivalent velocity dispersion values, $\sigma_{e}$ are shown in the $\mathrm{M}-\sigma_{e}$ plot in Fig. 1. Also plotted in the figure are the linear regression lines given by Tremaine et al. (2002), Ferrarese \& Merritt (2000), Gültekin et al. (2009) and McConnell \& Ma (2013) for late type galaxies (dashed, solid, dotted and long-dashed lines, respectively) for $M_{\mathrm{BH}}$ against $\sigma_{e}$. Most of our sample lie below the $\mathrm{M}-\sigma_{e}$ correlation. The probable reasons for the observed offset can be due the the effects of galaxy orientation in the measurement of $\sigma$ and/or the effects of bars in the increase of $\sigma$ in case of barred galaxies. We analysed 
the effects of any systematic bias in the $M_{\mathrm{BH}}$ estimates, the effects of galaxy orientation in the measurement of $\sigma$ and the increase of $\sigma$ due to the presence of bars and found that these effects are insufficient to explain the observed offset. The lack of bulge $-M_{\mathrm{BH}}$ co-evolution is also an indicative of secular evolution and is one of the probable reasons for the observed offset (Kormendy et al. 2013). A detailed study of the nature of the bulges and the role of dark matter in the growth of black holes in these systems are important.

\section{Discussion}

Seed BHs that are formed in the early universe grow by mass accretion to become the massive BHs that we observe in our local universe; the accretion is driven by galaxy mergers and interactions. LSB galaxies are usually isolated and lie close to the edge of voids. Simulations suggest that lighter seed BHs grow through slow accretion leading relatively low mass $\mathrm{BHs}$ and lie below the $M_{\mathrm{BH}}-\sigma_{e}$ correlation. These low mass and relatively pristine black holes can reveal important clues to the initial black hole mass function and help us to constrain the early evolution of black holes in galaxies. The black hole masses in our sample lie in the range $10^{5}-10^{7} \mathrm{M}_{\odot}$ and most of them lie below the $M_{\mathrm{BH}}-\sigma_{e}$ correlation. Also, their Eddington ratios are not high, hence their black holes are not accreting at a high rate and they fall in the low luminosity AGN (LLAGN) class. Thus, the BHs in GLSB galaxies represent one of the best candidates for pristine black holes and a good place to study seed black holes in our local universe.

\section{Acknowledgements}

The authors would like to thank the SDSS team for making the data publicly available. SS acknowledges the financial support from IAU for the participation in the symposium.

\section{References}

Baldwin J. A., Phillips M. M., \& Terlevich R., 1981, PASP, 93, 5

Bothun, G., Impey, C., \& McGaugh, S., 1997, PASP, 109, 745

Bothun, G. D., Schombert, J. M., Impey, C. D., Sprayberry, D., \& McGaugh, S. S. 1993, AJ, 106,530

Cappellari M., Emsellem E., 2004, PASP, 116, 138

Ferrarese L. \& Merritt D., 2000, ApJ, 539, L9

Gültekin, K., Richstone, D. O., Gebhardt, K. et al. 2009a, ApJ, 698, 198

Heckman, Timothy M., Kauffmann, Guinevere, Brinchmann, Jarle, Charlot, Stéphane, Tremonti, Christy, White, \& Simon D. M., 2004, ApJ, 613, 109

Impey, C. \& Bothun, Greg, 1997, ARAE A, 35, 2671

Impey, C., Burkholder, V., \& Sprayberry, D., 2001, AJ, 122, 2341

Impey, C. D., Sprayberry, D., Irwin, M. J., \& Bothun, G. D., 1996, ApJS, 105, 209

Jorgensen I., Franx M., \& Kjaergaard P., 1995, MNRAS, 276, 1341

Kormendy J. \& Ho L. C., 2013, ARA\&A, 51, 511

McConnell, Nicholas J. \& Ma, Chung-Pei, 2013, ApJ, 764, 184

Mei L., Yuan W., \& Dong X., 2009, Res. Astron. Astrophys., 9, 269

Morelli, L., Corsini, E. M., Pizzella, A., Dalla Bontá, E., Coccato, L., Méndez-Abreu, J., \& Cesetti, M., 2012, MNRAS, 423, 962

Pizzella A., Corsini E. M., Dalla Bontà E., Sarzi M., Coccato L., \& Bertola F., 2005, ApJ, 631, 785

Ramya, S., Prabhu, T. P., \& Das, M., 2011, MNRAS, 418, 789

Reines, Amy E., Greene, Jenny E., \& Geha, Marla, 2013, ApJ, 775, 116

Rosenbaum, S. D., Krusch, E., Bomans, D. J., \& Dettmar, R.-J., 2009, A\&SA, 504, 807 
Schombert, James M. \& Bothun, Gregory D., 1988, AJ, 95, 1389

Schombert, James M., Bothun, Gregory D., Schneider, Stephen E., \& McGaugh, Stacy S., 1992, $A J, 103,1107$

Silk, Joseph \& Rees, Martin J., 1998, A\&A, 331, 1

Sprayberry, D., Impey, C. D., Bothun, G. D., \& Irwin, M. J, 1995, AJ, 109, 558

Tremaine S. et al., 2002, ApJ, 574, 740

Volonteri, Marta, 2010, A\&ARv, 18, 279 